\title{
Dynamic Phase Signal Control Method for Unstable Asymmetric Traffic Flow at Intersections
}

\author{
Xiancai Jiang $\mathbb{D}$, Yu Jin $\mathbb{D}$, and Yanli Ma \\ School of Transportation Science and Engineering, Harbin Institute of Technology, Harbin 150001, China \\ Correspondence should be addressed to Xiancai Jiang; jxc023@126.com
}

Received 22 April 2020; Revised 7 December 2020; Accepted 7 January 2021; Published 22 February 2021

Academic Editor: Francesco Galante

Copyright (C) 2021 Xiancai Jiang et al. This is an open access article distributed under the Creative Commons Attribution License, which permits unrestricted use, distribution, and reproduction in any medium, provided the original work is properly cited.

\begin{abstract}
This paper addresses the limitations that the phases proposed in variable phase sequencing studies for stochastic traffic flow are all predetermined and that the variable phase sequencing is only suitable for low traffic volume environment. It presents a dynamic phase signal control method for unstable asymmetric traffic flow with two primary operational objectives: the realization of a dynamic phase scheme in each cycle and optimization of signal control parameters. First, an asymmetric state of traffic flow at signalized intersections is defined, rules governing the generation of the dynamic phase of each cycle based on asymmetric state are proposed, and the delay variations of intersections adopting dynamic phase schemes are modeled. Next, a signal control parameter adjustment algorithm for the dynamic phase is constructed to maximize the positive benefits of delay variation. Last, the operational performance of the proposed method is validated using data collected from an intersection in Harbin, China, by VISSIM simulation. Furthermore, it is found that a higher asymmetric coefficient leads to lower efficiency of a symmetrical release phase scheme at intersections, and the increase of average delay becomes significant when the asymmetric coefficient threshold is greater than 0.2 .
\end{abstract}

\section{Introduction}

Recently, the synchronization of traffic signal parameter optimization and network traffic flow equilibrium optimization has become a research hotspot within the field of traffic signal control. The deterministic and stochastic requirements [1], the minimum total travel time $[2,3]$, the equilibrium flow under uncertain travel demands and traffic delays [4], the equilibrium constraints under stochastic demands [5], and to ease traffic congestion [6,7] are all the focuses to be optimized. The purpose is to achieve the balanced utilization of road network resources. Although current traffic signal control research is trending toward networking [8] and intellectualization [9-13], for specific traffic scenarios, the referenced studies still cannot provide satisfactory solutions. One such scenario is asymmetric traffic flow at signalized intersections, which is still being studied.

Combinatorial phase models are one of the methods used to optimize traffic signal control for asymmetric traffic flow. Aimed at stable asymmetric traffic flow and unstable asymmetric traffic flow, there are two applicable scenarios for this method. The first scenario typically adopts a fixed cycle time and overlapping phases [14], while the second scenario usually uses an adaptive signal control strategy to optimize the signal control parameters. Research methods focus on an adaptive signal control strategy which includes an integrated optimization approach [15], implementation of RHODES (Real-time, Hierarchical, Optimized, Distributed, and Effective System) on simulation models of actual scenarios [16], the Markovian decision process [17], the dynamic gap-out approach [18], and a multistage stochastic program based on phase clearance reliability (PCR) [19], among others. However, the adaptive signal control strategy only optimizes the signal control parameters, while the phase scheme remains unchanged.

To address the limitations of adaptive signal control strategies, variable phase sequencing has been introduced into traffic signal optimization control in various studies [20-25]. Approximate dynamic programming (ADP) [20], 
colored Petri nets (CPN) [21], modified job scheduling algorithms [22, 23], forward search algorithms based on dynamic programming (FSDP) [24], and fuzzy control algorithms [25] are among the methods proposed to optimize the signal control parameters of the variable phase sequence. However, all the phases are predetermined, and these methods are only applicable to low traffic volume intersections.

Dynamic lane setting is another method used to optimize traffic signal control for asymmetric traffic flow. In recent years, research on dynamic lane setting has focused on dynamic lane assignment [26-29], optimization of the signal phase [30-35], and operational efficiency evaluations [36-38]. By integrating the optimization of lane group space allocation with the optimization of signal timing parameters, dynamic lane setting can improve the mobility of the entire intersection [31, 34, 35].

The presented literature primarily address the optimization of stochastic arrival traffic flow in low traffic volume environments and the stable asymmetric traffic flow of opposite approaches at signalized intersections, e.g., the east and west approaches defined as a pair of opposite approaches. But, the effect of opposite traffic flow unstable asymmetry on the benefits of signal control schemes in each cycle is ignored, when the hourly traffic volume of the opposite approaches is essentially symmetrical. To deal with this issue, some researchers believe that the signal control scheme of symmetrical phases can be adjusted and solved by itself [39]. This is primarily based on the hypothesis of traffic signal control theory, which postulates that the arrival of traffic flow at each approach is uniform or obeys a certain distribution $[14,25,39]$. Under this hypothesis, the asymmetry of opposite traffic flow is generally slight in each cycle, so the symmetrical phase is valid. However, when the asymmetry is severe and unstable, it will cause the unbalanced use of intersection space-time resources and an increase in stops and delays. At present, the phase optimization method for unstable asymmetric traffic flow is still unaddressed.

The presented literature has a common characteristic: the phases are predetermined. Compared to the existing work, the novel contributions of our work are as follows:

(1) Rules governing the generation of the dynamic phase of each cycle based on unstable asymmetric traffic flow are proposed

(2) The delay variations of intersections adopting dynamic phase schemes are modeled

(3) A signal control parameter adjustment algorithm for the dynamic phase is constructed with the objective of maximizing the comprehensive traffic benefits

The remainder of this paper is organized as follows. Section 2 presents the optimization methodology for unstable asymmetric traffic flow. A traffic simulation environment is established in Section 3, and the empirical studies are conducted in Section 4. Finally, some conclusions and future research are discussed in Section 5.

\section{Methodology}

2.1. Definition of Asymmetric Traffic Flow. To any pair of opposite approaches at an intersection, such as the east and west approaches in a four-arm intersection, as shown in Figure 1, the opposite traffic commonly includes opposite left-turn traffic flow, through traffic flow and right-turn traffic flow. Because right-turn vehicles are usually released at the same time with the through vehicles in the same approach, this paper takes the right-turn traffic flow and the through traffic flow in the same approach as a whole. Due to the traffic conflict between the left-turn traffic flow and the through traffic flow for the opposite approaches in signal control, these traffic flows need to be listed separately for phase design and optimization of signal control parameters. The numbering rules of opposite traffic flow in this paper are shown in Figure 1.

The difference in per lane traffic volume to be released in a cycle for the opposite approaches can reflect whether the opposite traffic flow is symmetrical. The larger the difference, the more asymmetric the opposite traffic flow is. Therefore, the asymmetric coefficient of opposite traffic flow is defined as

$$
a c_{i}=\frac{\left(q_{i, 1} / n_{i, 1}\right)-\left(q_{i, 2} / n_{i, 2}\right)}{\max \left(\left(q_{i, 1} / n_{i, 1}\right),\left(q_{i, 2} / n_{i, 2}\right)\right)},
$$

where $a c_{i}$ is the asymmetric coefficient of the $i^{\text {th }}$ phase, $q_{i, j}$ is the traffic volume in the $j^{\text {th }}$ approach of the $i^{\text {th }}$ phase (pcu), and $n_{i, j}$ represents the number of lanes in the $j^{\text {th }}$ approach of the $i^{\text {th }}$ phase.

As can be seen from equation (1), the asymmetric coefficient of opposite traffic flow could be either positive or negative. If $\left(q_{i, 1} / n_{i, 1}\right)-\left(q_{i, 2} / n_{i, 2}\right) \geq 0$, the asymmetric coefficient is positive; otherwise, it is negative.

The opposite traffic flow is symmetrical or asymmetrical depends on whether the asymmetric coefficient of the opposite traffic flow exceeds the threshold value? The symmetry state of the phase of traffic flow is defined as

$$
p s_{i}= \begin{cases}1, & a c_{i}>0, \\ & a c_{i} \geq a c_{i, 0}, \\ 0, & \left|a c_{i}\right|<a c_{i, 0}, \\ -1, & a c_{i}<0, \\ & \left|a c_{i}\right| \geq a c_{i, 0} .\end{cases}
$$

Here, $p s_{i}$ is the symmetry state of the $i^{\text {th }}$ phase, and $a c_{i, 0}$ is the threshold value. The threshold value of each intersection can be obtained by traffic simulation. In this paper, $a c_{i, 0}=0.2$ is reasonable, and the basis for selecting this threshold is shown in Section 4.2.2. $p s_{i}=0$ indicates that the opposite traffic flow of the $i^{\text {th }}$ phase is suitable to be released symmetrically in a cycle, and $p s_{i} \neq 0$ indicates that the symmetrical release is not selected prior to the opposite traffic flow of the $i^{\text {th }}$ phase. The positive or negative value shows the asymmetric direction of the $i^{\text {th }}$ phase traffic flow. 


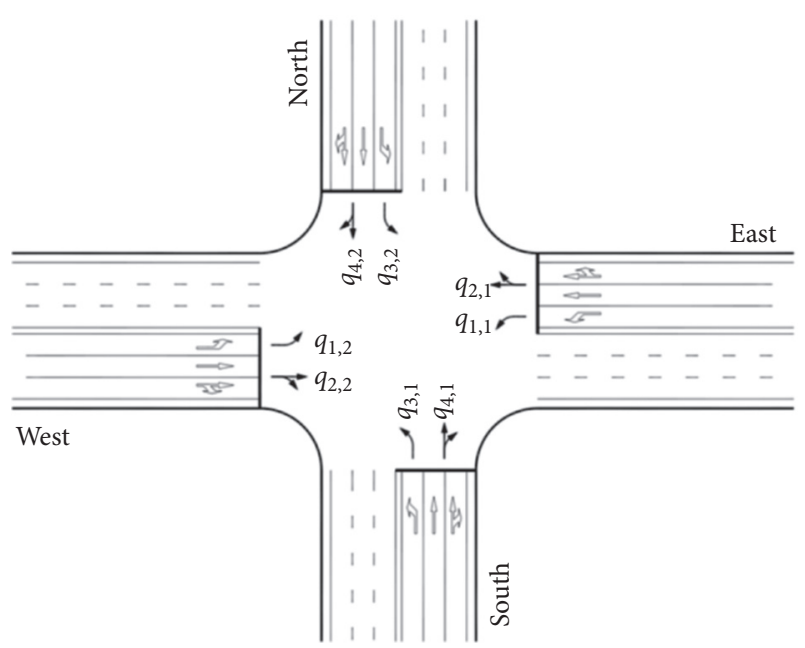

FIgURE 1: Example of an intersection layout.

2.2. Detector Location and Stochastic Traffic Volume Collection. Two sets of detectors are placed on each approach at the intersection to collect the stochastic traffic volume per cycle in all approaches, as shown in Figure 2.

Detector 1 is used to decide whether the saturation traffic flow disappears during the green time, and detector 2 is used to detect the number of arrival vehicles. The location of detector 1 is chosen based on two factors. The first factor ensures that the last saturated vehicle detected by detector 1 can pass the stop line smoothly in the yellow time. That is, at the end of the green time, there will be no queuing vehicles before detector 1 . The farthest distance from the stop line is given by

$$
L_{i, j}^{d}=v_{i, j} Y_{i}
$$

where $L_{i, j}^{d}$ is the farthest distance from the stop line in the $j^{\text {th }}$ approach of the $i^{\text {th }}$ phase from which the vehicle can pass the stop line smoothly in the yellow time $(\mathrm{m}), v_{i, j}$ is the mean speed of vehicles in the $j^{\text {th }}$ approach of the $i^{\text {th }}$ phase $(\mathrm{m} / \mathrm{s})$, and $Y_{i}$ is the yellow time of the $i^{\text {th }}$ phase $(s)$.

The second factor ensures that detector 1 will be able to detect the saturation traffic flow. Considering the queue length per cycle, the location of detector 1 needs to be less than the queue length. The vehicle queue length is given by

$$
L_{i, j}^{q}=\frac{h_{i, j}^{s} a_{i, j}\left(1-\lambda_{i}\right) c}{n_{i, j}},
$$

where $L_{i, j}^{q}$ is the queue length in the $j^{\text {th }}$ approach of the $i^{\text {th }}$ phase $(\mathrm{m}), h_{i, j}^{s}$ is the queuing head spacing in the $j^{\text {th }}$ approach of the $i$ th phase $(\mathrm{m})$, and $a_{i, j}$ is the vehicle arrival rate in the $j^{\text {th }}$ approach of the $i^{\text {th }}$ phase ( $\left.\mathrm{pcu} / \mathrm{s}\right)$. The cycle time is $c$ (s), and $\lambda_{i}$ denotes the green split of the $i^{\text {th }}$ phase.

Owing to the randomness of the arrival vehicles, half of the queue length is used as the location of detector 1 based on 45 signalized intersections and 4,520 groups of survey data on queuing length per cycle in Harbin, China. The minimum values of equations (3) and (4) are used as the final location of detector 1 , as given by

$$
L_{i, j}^{d_{1}}=\min \left(L_{i, j}^{d}, 0.5 L_{i, j}^{q}\right),
$$

where $L_{i, j}^{d_{1}}$ is the distance between detector 1 and the stop line in the $j^{\mathrm{th}}$ approach of the $i^{\text {th }}$ phase $(\mathrm{m})$.

Meanwhile, in order to detect the number of arrival vehicles for each phase of the approach in a cycle, the location of detector 2 has to be larger than the queue length of the vehicles and be located in the section where the vehicles have completed lane changing. Owing to the stochastic nature of the arrival vehicles, the location of detector 2 is taken as 1.5 times the queue length based on the same survey data of queuing length per cycle at signalized intersections in Harbin, China, as given by

$$
L_{i, j}^{d_{2}}=1.5 L_{i, j}^{q}
$$

where $L_{i, j}^{d_{2}}$ is the distance between detector 2 and the stop line in the $j^{\mathrm{th}^{j}}$ approach of the $i^{\text {th }}$ phase $(\mathrm{m})$.

In order to adapt to the queue length changes at different times of the day, the queue length of the peak hour is used to determine the locations of the detectors. Then, the stochastic traffic volume to be processed in each approach of each phase per cycle is given by

$$
q_{i, j, k}=q_{i, j, k}^{d_{2}}+q_{i, j, k-1}^{d_{2}}-q_{i, j, k-1}^{d_{1}},
$$

where $q_{i, j, k}$ is the traffic volume in the $j^{\text {th }}$ approach of the $i^{\text {th }}$ phase of the $k^{\text {th }}$ cycle (pcu), $q_{i, j, k}^{d_{2}}$ is the number of arriving vehicles detected by detector 2 in the $j^{\text {th }}$ approach of the $i^{\text {th }}$ phase of the $k^{\text {th }}$ cycle ( $\mathrm{pcu}$ ), and $q_{i, j, k-1}^{d_{1}}$ is the number of leaving vehicles detected by detector 1 in the $j^{\text {th }}$ approach of the $i^{\text {th }}$ phase of the $(k-1)^{\text {th }}$ cycle $(\mathrm{pcu})$.

\subsection{Phase Optimization per Cycle Based on the Unstable Traffic} Flow Distribution. How to optimize the traffic signal scheme for the unstable arrival traffic volume in view of the cycle? Some researchers have adopted the adaptive signal control strategy and variable phase sequence to address the issue [18-20], though without considering the distribution states of opposite traffic flow and without proposing the adaptive phase solution.

2.3.1. Phase Scheme Applicable to the Distribution of Opposite Traffic Flow. Taking the east-west approaches of the intersection as an example, according to equation (2), the opposite traffic flow can exhibit the following four states, as shown in Figure 3.

(1) Both the left-turn and through traffic flows are symmetrical, namely, $p s_{1}=0$ and $p s_{2}=0$, as seen in Figure 3(a). In this case, the symmetrical phase can make full use of the green time.

(2) The left-turn traffic flow is asymmetrical, and the through traffic flow is symmetrical, or vice versa, i.e., $p s_{1} \times p s_{2}=0$ and $p s_{1} \neq 0$ or $p s_{2} \neq 0$, as seen in Figure 3(b). Considering $p s_{1} \neq 0$ as the example, the symmetrical phase can make full use of the green time of the through phase, but there would be some time when there are no vehicles in one approach of 


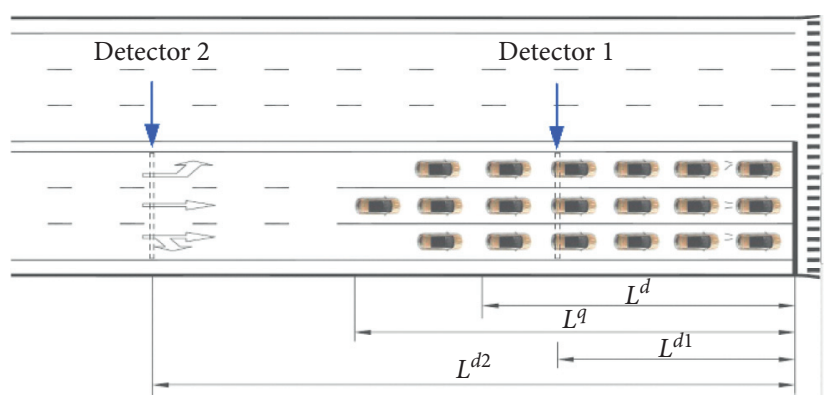

FIGURE 2: Schematic of detector locations.

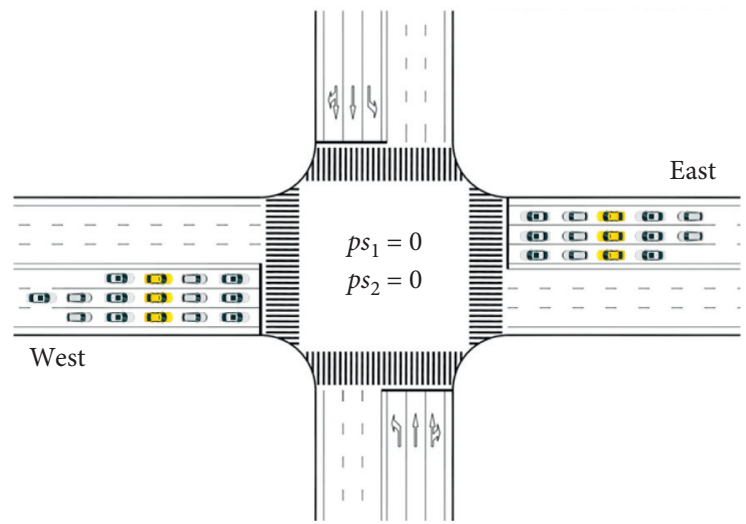

(a)

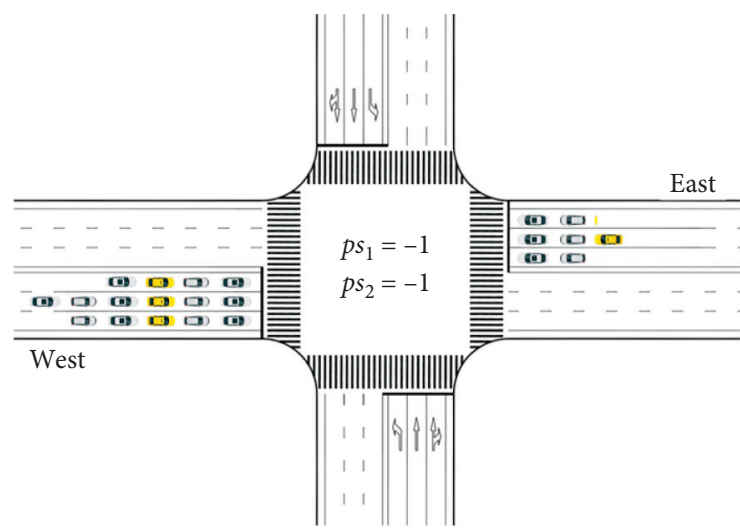

(c)

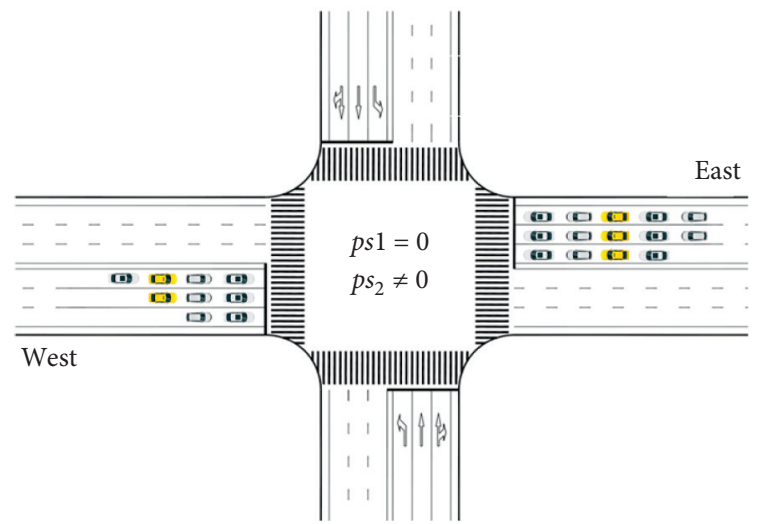

(b)

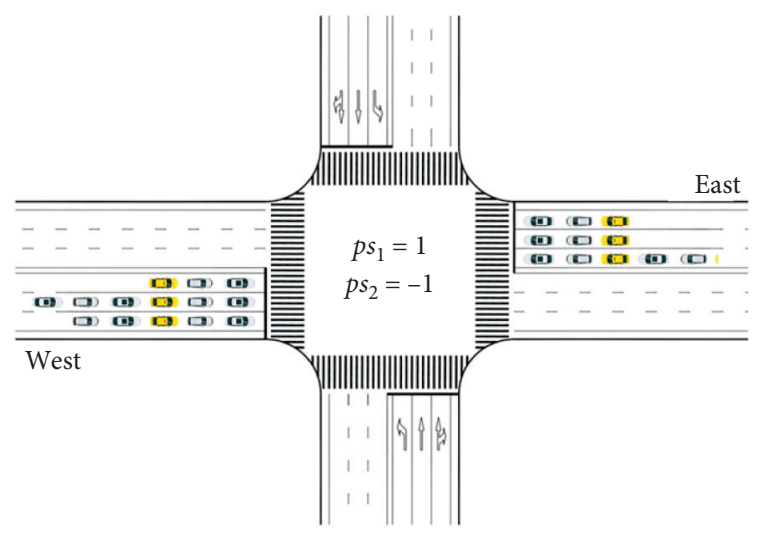

(d)

Figure 3: Possible states of opposite traffic flow in the east and west approaches when $a c_{i, 0}=0.2$. (a) Both left-turn and through traffic flows are symmetrical. (b) Left-turn traffic flow is asymmetrical and through traffic flow is symmetrical, or vice versa. (c) Left-turn traffic flow is asymmetrical in the same direction to through traffic flow. (d) Left-turn traffic flow is asymmetrical in the opposite direction to through traffic flow.

the left-turn traffic flow and the light is still green. If the left-turn, through, and right-turn traffic flows of each approach are arranged to a dedicated phase to be released, i.e., a phase for each approach, there will be an uneven utilization of the left-turn and through lanes. Therefore, in order to achieve improved signal control benefits, the better phase scheme depends on which scheme the sum of the ratio of traffic volume to saturation flow rate is smaller, when a symmetrical phase or a phase for each approach is adopted.

(3) The left-turn traffic flow is asymmetrical in the same direction to through traffic flow, namely, $p s_{1} \times p s_{2}=1$, as seen in Figure 3(c). In this case, the symmetrical phase leads to a low utilization rate of the green time on approaches with low traffic volumes. If a phase for each approach is adopted, there will also be an uneven utilization of the leftturn and through lanes. However, if the left-turn traffic flow is released symmetrically first, when the saturated traffic flow disappears in the approach with a small traffic volume, the left-turn green light can be turned off early, which will allow the opposite through traffic flow to pass together with the left-turn vehicles. 
In this case, the delay is used to evaluate the benefits obtained by extending the green time, $\Delta t_{1}$, of the leftturn phase and the one obtained by immediately ending the left-turn phase. The end time of the leftturn phase is dependent on when the net of those benefits is less than 0 . After the left-turn phase is complete, the east-west through phase functions until the saturated traffic flow of the through vehicles disappears. In this way, the green time and approach lanes can be fully utilized.

(4) The left-turn traffic flow is asymmetrical in the opposite direction to through traffic flow, namely, $p s_{1} \times p s_{2}=-1$, as seen in Figure 3(d). To reduce the green time loss, the symmetrical phase is considered. The green time of each phase only meets the mean traffic volume in each approach, and the stranded vehicles are arranged to be released in the next cycle.

To deal with asymmetric traffic flow, Akcelik (1995) proposed a diamond turn-type phasing arrangement [39]. This method is only applicable to the asymmetric traffic flow state where $p s_{1} \times p s_{2}=1$ or $p s_{3} \times p s_{4}=1$, which is only one of many asymmetric traffic states. Therefore, this method is difficult to apply to other asymmetric traffic flow states. However, it is often used in stable asymmetric traffic flow to improve the signal control efficiency at intersections [31,32]. Additionally, the standard NEMA ring barrier structure can also deal with stable asymmetric traffic flow.

\subsubsection{Generation Rule of Dynamic Phase in Each Cycle.} The signalized intersection utilizes the following rules to produce the dynamic phase according to the unstable distribution state of opposite traffic flow per cycle. The eastwest approaches are used as the example, and the rules for the north-south approaches are similar.

(1) When $p s_{1}=0$ and $p s_{2}=0$, the left-turn and through traffic flows are released symmetrically, respectively. The symmetrical phase is adopted.

(2) When $p s_{1} \times p s_{2}=0$, but $p s_{1} \neq 0$ or $p s_{2} \neq 0$, if $[(\max$ $\left.\left.\left(q_{1,1}, q_{1,2}\right) / s_{1}\right)+\left(\max \left(q_{2,1}, q 2,2\right) / s_{2}\right)\right]<\left[\max \left(\left(q_{1,1} /\right.\right.\right.$ $\left.\left.\left.s_{1}\right),\left(q_{2,1} / s_{2}\right)\right)+\max \left(\left(q_{1,2} / s_{1}\right),\left(q_{2,2} / s_{2}\right)\right)\right]$, the symmetrical phase is adopted. Otherwise, a phase for each approach is adopted. Here, $s_{i}$ is the saturation flow rate of the $i^{\text {th }}$ phase when the traffic flow is released symmetrically (pcu/s). For example, $s_{1}$ refers to the saturation flow rate of the east-west leftturn lanes when the left-turn traffic flow of the east and west approaches is first released at the same time.

(3) When $p s_{1} \times p s_{2}=1$, the left-turn traffic flow is symmetrically released first, and the left-turn signal light of the approach with small traffic volume is closed until the saturated traffic flow disappears. Then, the left-turn and through traffic flows on the same approach are released, with the releasing time dependent on when the net of benefit evaluation is less than 0, as seen in Section 2.4. Finally, the through traffic flow is symmetrically released until the saturated traffic flow disappears, and then, the through phase is turned off.

(4) When $p s_{1} \times p s_{2}=-1$, the symmetrical phases for the left-turn traffic flow and the through traffic flow are adopted, respectively. The green time of left-turn phase is determined by the traffic volume $\left(q_{1,1}+q_{1,2}\right) / 2$, and the one of through phase is determined by the traffic volume $\left(q_{2,1}+q_{2,2}\right) / 2$.

\subsection{Benefit Evaluation of Extending the ${ }^{\text {th }}$ Phase Green Time by $\Delta t_{i}$}

(1) First, we consider the delay change when the overlapping phase is used, taking the left-turn phase of the east-west approach as an example.

Figure 4(a) shows the delay variation when the leftturn traffic flow is not symmetrical and the through traffic flow in one approach overlaps the left-turn traffic flow. The area of quadrilateral $B C E D$ represents the delay in through traffic flow that should have occurred if the dynamic phase scheme for asymmetric traffic flow is not implemented. Figure 4(b) shows the delay evolution in the subsequent arrival of left-turn vehicles due to early closure of the traffic light in the approach of low volume left-turn arrivals, i.e., the area of $A B C D$ in Figure 4(b).

After implementation of the dynamic phase scheme, the reduced delay of the through phase in the eastwest approach is given by

$$
\Delta d_{1}= \begin{cases}\frac{q_{2,2} \Delta t_{1}\left(2 r_{2}-\Delta t_{1}\right)}{2\left(1-\left(q_{2,2} / s_{2}\right)\right)}, & q_{1,1}<q_{1,2}, \\ \frac{q_{2,1} \Delta t_{1}\left(2 r_{2}-\Delta t_{1}\right)}{2\left(1-\left(q_{2,1} / s_{2}\right)\right)}, & q_{1,1}>q_{1,2},\end{cases}
$$

where $\Delta t_{i}$ is the extended green time of the $i^{\text {th }}$ phase (s) and $r_{i}$ is the red time of the $i^{\text {th }}$ phase (s).

The increased delay in the subsequent arrival of leftturn vehicles due to early closure of the traffic signal in the approach of low volume left-turn arrivals can be expressed by

$$
\Delta d_{2}=\frac{\min \left(q_{1,1}, q_{1,2}\right) \Delta t_{1}\left(2 r_{1}+\Delta t_{1}\right)}{2\left[1-\left(\min \left(q_{1,1}, q_{1,2}\right) / s_{1}\right)\right]} .
$$

The total delay variations are given by

$$
\Delta D_{1,1}=\Delta d_{2}-\Delta d_{1},
$$

where $\Delta D_{i, 1}$ is the variation benefits of the $i^{\text {th }}$ phase when the $i^{\text {th }}$ phase extends the green time by $\Delta t_{i}(\mathrm{~s})$. The evolution of $\Delta D_{1,1}$ following $\Delta t_{1}$ is analyzed. Assuming $\Delta q_{1,1}<\Delta q_{1,2}$ (analysis process for $\Delta q_{1,1}>$ $\Delta q_{1,2}$ is the same) and substituting equations (8) and (9) into equation (10), we get the following equation: 


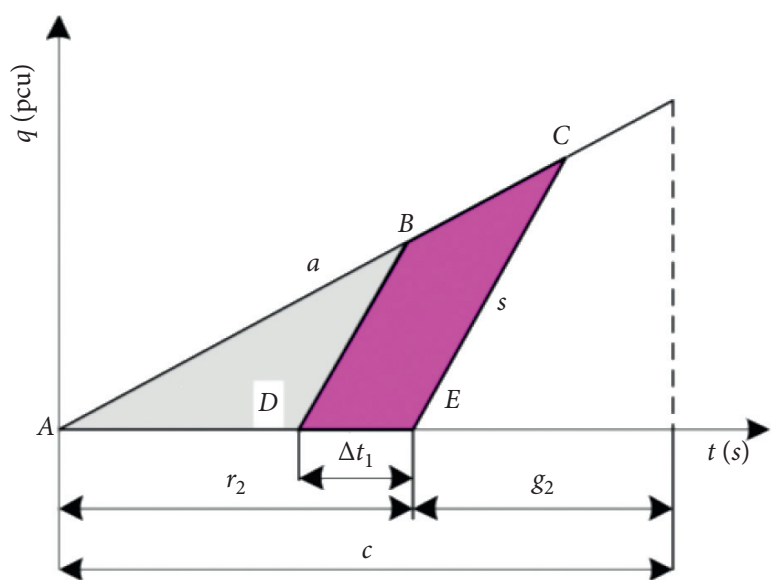

(a)

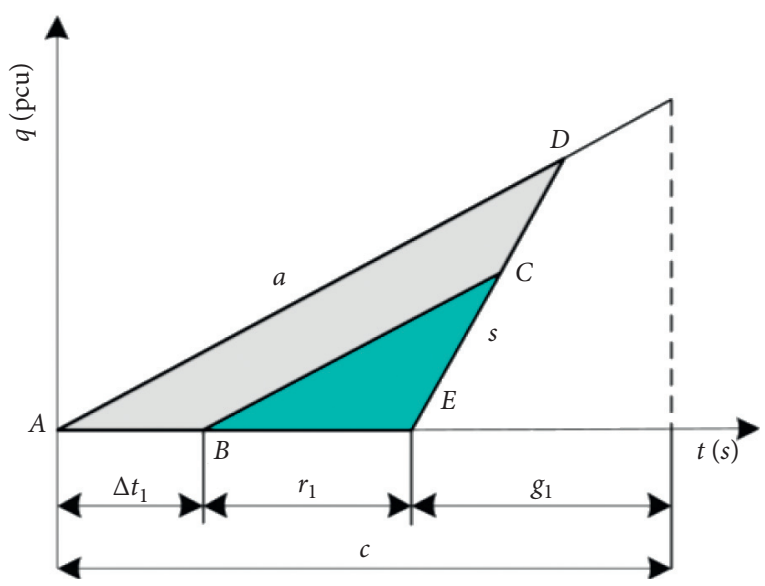

(b)

Figure 4: Delay variations when an overlapping phase is used. (a) Reduced delay of the through phase. (b) Increased delay in the approach of low volume left-turn arrivals.

$$
\Delta D_{1,1}=-\left(\frac{q_{2,2}}{2\left(1-\left(q_{2,2} / s_{2}\right)\right)}+\frac{q_{1,1}}{2\left(1-\left(q_{1,1} / s_{1}\right)\right)}\right) \Delta t_{1}^{2}+\left(\frac{r_{2} q_{2,2}}{1-\left(q_{2,2} / s_{2}\right)}+\frac{r_{1} q_{1,1}}{1-\left(q_{1,1} / s_{1}\right)}\right) \Delta t_{1} .
$$

Equation (11) is a quadratic equation of one variable with a downward opening. That is to say, early termination of left-turn green time in the approach with small traffic volume will not offset the increased delays in the subsequent arrival of left-turn vehicles by reducing the delays of through vehicles.

(2) Next, we incorporate the comprehensive benefits of extending the current phase green time by $\Delta t_{1}$.

Taking the extension of the left-turn phase in the east-west approach as an example, when the saturated traffic flow disappears in one approach of the left-turn phase (assuming the east approach first disappears), the comprehensive benefits of extending the green time by $\Delta t_{1}$ in the other approach consists of two parts. First is the reduced equivalent delay of the departing vehicles of the left-turn phase due to not having to wait for red lights within a time interval of $\Delta t_{1}$, which is a positive benefit. The second part is the increased delay to the other phases' queuing vehicles caused by the extra waiting time of $\Delta t_{1}$ and the increased equivalent delay of the newly arriving vehicles of other phases within a time interval of $\Delta t_{1}$, which is a negative benefit.

$$
\begin{aligned}
\Delta D_{1,2}= & r_{1} K_{1}+f_{1} K_{1}-\Delta t_{1} N-f_{1} M \\
r_{1}= & Y_{1}+Y_{2}+\frac{\left\{\max \left[N_{2,1}(0)+q_{2,1} \Delta t_{1}-s_{2} \Delta t_{1}, N_{2,2}(0)+q_{2,2} \Delta t_{1}\right]\right\}}{\left[s_{2}-\max \left(q_{2,1}, q_{2,2}\right)\right]} \\
& +Y_{3}+\frac{\left\{\max \left[N_{3,1}(0), N_{3,2}(0)\right]+\max \left(q_{3,1}, q_{3,2}\right) \Delta t_{1}\right\}}{\left[s_{3}-\max \left(q_{3,1}, q_{3,2}\right)\right]} \\
& +Y_{4}+\frac{\left\{\max \left[N_{4,1}(0), N_{4,2}(0)\right]+\max \left(q_{4,1}, q_{4,2}\right) \Delta t_{1}\right\}}{\left[s_{4}-\max \left(q_{4,1}, q_{4,2}\right)\right]}, \\
K_{1}= & s_{1} \Delta t_{1}, \quad \\
N= & N_{2,1}(0)+N_{2,2}(0)+q_{2,2} \Delta t_{1}+N_{3,1}(0)+N_{3,2}(0)+\left(q_{3,1}+q_{3,2}\right) \Delta t_{1} \\
& +N_{4,1}(0)+N_{4,2}(0)+\left(q_{4,1}+q_{4,2}\right) \Delta t_{1}, \\
M= & q_{2,2} \Delta t_{1}+\left(q_{3,1}+q_{3,2}\right) \Delta t_{1}+\left(q_{4,1}+q_{4,2}\right) \Delta t_{1},
\end{aligned}
$$


where $\Delta D_{i, 2}$ is the variation benefits of other phases when the $i^{\text {th }}$ phase extends the green time by $\Delta t_{i}(\mathrm{~s})$, $N$ is the total number of queuing vehicles (pcu), and $M$ is the total arriving vehicles from other phases when the $i^{\text {th }}$ phase extends the green time by $\Delta t_{i}$ (pcu). $K_{i}$ represents the number of vehicles passing through the stop line at the approach of the $i^{\text {th }}$ phase with a large traffic volume within a time interval $\Delta t_{i}$ (pcu). The number of queuing vehicles in the $j^{\text {th }}$ approach of the $i^{\text {th }}$ phase is denoted by $N_{i, j}(0)(\mathrm{pcu})$, and $f_{i}$ is the equivalent coefficient for a stop.

(3) Finally, we calculate the total benefits.

The total benefits when the current phase extends the green time by $\Delta t_{1}$ are given by

$$
D_{1}=\Delta D_{1,1}+\Delta D_{1,2} \text {. }
$$

It is noted that this extension is only meaningful when $D_{1} \geq 0$.

2.5. Signal Control Parameter Adjustment Algorithm. According to the generation rule of the dynamic phase in each cycle given in Section 2.3.2, the solution process for the signal control parameters is as follows:

(1) When the state of opposite traffic flow meets rules (1) and (2),

If the symmetrical phase is adopted, then green time of each phase is determined by

$$
g_{i, k}=\frac{\max \left(q_{i, 1, k}, q_{i, 2, k}\right)}{s_{i}}+l,
$$

where $g_{i, k}$ is the green time of the $i^{\text {th }}$ phase of the $k^{\text {th }}$ cycle (s) and $l$ is the set-up loss time (s).

If a phase for each approach is adopted, then the green time of each phase is determined by

$$
g_{i, k}= \begin{cases}\frac{q_{i, 1, k}+q_{i+1,1, k}}{s_{i}^{\prime}}+l, & i=1,3, \\ \frac{q_{i, 2, k}+q_{i-1,2, k}}{s_{i}^{\prime}}+l, & i=2,4,\end{cases}
$$

where $s_{i}^{\prime}$ is the saturation flow rate of the $i^{\text {th }}$ phase when the traffic flow of an approach is released alone $(\mathrm{pcu} / \mathrm{s})$. For example, $s_{1}^{\prime}$ refers to the saturation flow rate of all left-turn, through, and right-turn lanes of the east approach when the traffic flow of the leftturns, through, and right-turns at the east approach is first released at the same time.

(2) When the state of opposite traffic flow meets rule (3),

$$
g_{i, k}=\frac{\min \left(q_{i, 1, k}, q_{i, 2, k}\right)}{s_{i}}+l .
$$

As long as the total benefits are positive, the longer the extended green time, $\Delta t_{i}$, of the $i^{\text {th }}$ phase, the better the traffic benefits when the overlapping phase is adopted. Therefore, the optimization objective can be expressed by

$$
\max (\Delta T)=\max \sum_{i=1}^{p} \Delta t_{i}
$$

(3) When the state of opposite traffic flow meets rule (4), The green time of each phase is determined by

$$
g_{i, k}=\frac{q_{i, 1, k}+q_{i, 2, k}}{2 s_{i}} .
$$

The constraint conditions are

$$
\left\{\begin{array}{l}
D_{i} \geq 0, \quad i=1,2, \ldots, p, \\
c_{k}=\sum g_{i, k}+L, \\
g_{i, \min } \leq g_{i, k} \leq g_{i, \max },
\end{array}\right.
$$

where $L$ is the total loss time per cycle $(\mathrm{s}), g_{i, \min }$ is the minimum green time of the $i^{\text {th }}$ phase $(\mathrm{s})$, and $g_{i, \max }$ is the maximum green time of the $i^{\text {th }}$ phase (s). The cycle time of the $k^{\text {th }}$ cycle is $c_{k}(\mathrm{~s})$, and $p$ represents the number of phases.

2.6. Solution Algorithms. As $\Delta t_{i}$ is usually an integer greater than or equal to one and the maximum extension times, $\Delta t_{i}$, of each phase are not correlated, an exhaustive method can be used to solve the optimization problem of equation (17). The solution algorithm is given as follows:

Step 1: let $i=1$ and $\Delta t_{i}=0$.

Step 2: let $\Delta t_{i}=\Delta t_{i}+1$, and $D_{i}$ is calculated.

Step 3: if $D_{i}>0$, then return Step 2; otherwise, $g_{i, k}=g_{i, k}+\Delta t_{i}-1$, let $i=i+1$, and $\Delta t_{i}=0$.

Step 4: if $i \leq 4$, then return Step 2; otherwise, end the solution process. Under all constraints, the final values of $g_{i, k}$ are determined.

\section{Case Study}

3.1. Statistical Analysis of Asymmetric Traffic Flow at Signalized Intersections. Forty-five signalized intersections and 4,520 groups of survey data for queuing length per cycle in Harbin, China, are listed in Table 1. Symmetrical release is adopted at all investigated intersections.

As seen in Table 1, regardless of the time, the asymmetric phenomenon of opposite traffic flow existed from the perspective of the signal cycle, particularly in the off-peak hours. In all of the asymmetric cycles, the asymmetric direction of opposite traffic flow is not the same, indicating an alternating state. These results show that a symmetrical release at these intersections may not achieve the best signal control benefits.

3.2. Basic Conditions of the Simulation Intersection. The intersection of Hongqi Street and Huaihe Road in Harbin, China, is utilized as an example, as seen in Figure 5. The lane division, traffic volume of each approach in the peak hour, and fixed signal timing parameters currently in operation are shown in Table 2. In the peak hour traffic volume row in 
TABle 1: Distribution of asymmetric traffic flow $\left(a c_{i, 0}=0.2\right)$.

\begin{tabular}{lccccc}
\hline Time & Total cycles & Asymmetric cycles & $p s_{i}=1$ & $p s_{i}=-1$ & Rate $(\%)$ \\
\hline Peak hours & 2287 & 759 & 422 & 337 & 33.19 \\
Off-peak hours & 2233 & 1352 & 627 & 725 & 60.55 \\
\hline
\end{tabular}

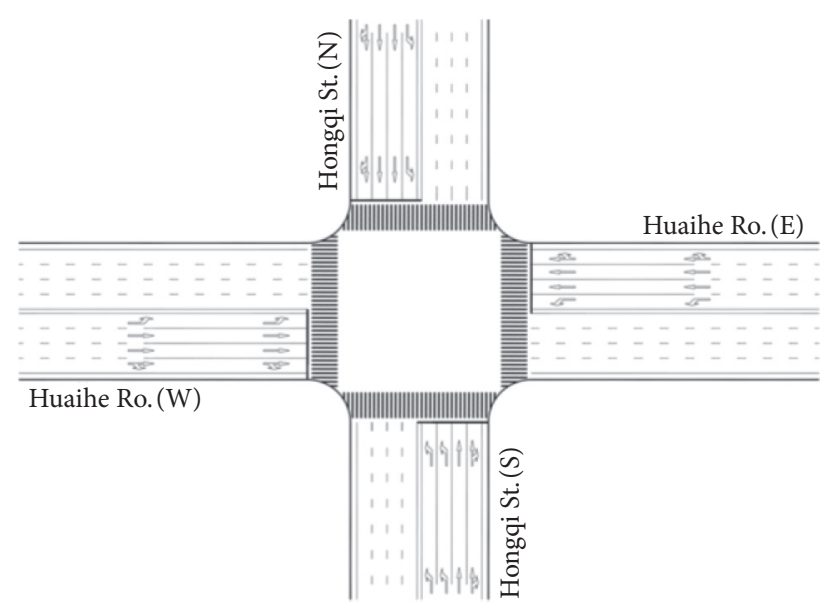

FIgURE 5: The intersection layout of Hongqi Street and Huaihe Road.

Table 2: Conditions of the Hongqi Street and Huaihe Road intersection.

\begin{tabular}{|c|c|c|c|c|c|c|c|c|}
\hline \multirow[b]{2}{*}{ Items } & \multicolumn{8}{|c|}{ Flow } \\
\hline & $\begin{array}{l}\text { North left- } \\
\text { turn }\end{array}$ & $\begin{array}{l}\text { South left- } \\
\text { turn }\end{array}$ & $\begin{array}{l}\text { North through } \\
\text { (right-turn) }\end{array}$ & $\begin{array}{l}\text { South through } \\
\text { (right-turn) }\end{array}$ & $\begin{array}{l}\text { West left- } \\
\text { turn }\end{array}$ & $\begin{array}{c}\text { East left- } \\
\text { turn }\end{array}$ & $\begin{array}{l}\text { West } \\
\text { through } \\
\text { (right- } \\
\text { turn) }\end{array}$ & $\begin{array}{l}\text { East through } \\
\text { (right-turn) }\end{array}$ \\
\hline Phase scheme & & 16 & & & Z & & & $\stackrel{\leftrightarrow}{\leftrightarrow}$ \\
\hline Pedestrian phase & \multicolumn{4}{|c|}{ South-north release } & \multicolumn{4}{|c|}{ East-west release } \\
\hline $\begin{array}{l}\text { Peak hour traffic } \\
\text { volume }(\mathrm{pcu} / \mathrm{h})\end{array}$ & 288 & 320 & $1,295(289)$ & $1,076(132)$ & 218 & 230 & $757(83)$ & $773(265)$ \\
\hline Green time (s) & \multicolumn{2}{|c|}{24} & \multicolumn{2}{|c|}{43} & \multicolumn{2}{|c|}{24} & \multicolumn{2}{|r|}{29} \\
\hline Yellow time (s) & \multicolumn{2}{|c|}{4} & \multicolumn{2}{|c|}{4} & \multicolumn{2}{|c|}{4} & \multicolumn{2}{|r|}{4} \\
\hline Red time (s) & \multicolumn{2}{|c|}{107} & \multicolumn{2}{|c|}{88} & \multicolumn{2}{|c|}{107} & \multicolumn{2}{|r|}{102} \\
\hline Green ratio & \multicolumn{2}{|c|}{0.19} & \multicolumn{2}{|c|}{0.33} & \multicolumn{2}{|c|}{0.19} & \multicolumn{2}{|r|}{0.22} \\
\hline$L^{q}(\mathrm{~m})$ & 53 & 29 & 80 & 92 & 40 & 42 & 49 & 61 \\
\hline$L^{d}(\mathrm{~m})$ & 40 & 40 & 40 & 40 & 40 & 40 & 40 & 40 \\
\hline$L^{d_{1}}(\mathrm{~m})$ & 26 & 15 & 40 & 40 & 20 & 21 & 25 & 31 \\
\hline$L^{d_{2}}(\mathrm{~m})$ & 79 & 44 & 120 & 138 & 60 & 63 & 74 & 92 \\
\hline
\end{tabular}

Table 2, the figures between the brackets represent the rightturn hourly traffic volume.

This particular intersection is chosen as a case study owing to the hourly through traffic volume of the east and west approaches being similar and the opposite traffic flow in the majority of cycles being alternately asymmetric. In China, the right-turn movement can also pass during the red time. Although the right-turn traffic volume of the east approach is $182 \mathrm{pcu} / \mathrm{h}$ greater than that of the west approach, the total number of queuing vehicles in the east and west approaches is very close in the peak hour.

Survey results show that the average queuing head spacing is $6.5 \mathrm{~m}$, and the mean speed is $36 \mathrm{~km} / \mathrm{h}$ at the intersection. According to these conditions, the locations of the detectors on each approach are calculated by equations (3)-(6), which have also been included in Table 2 .

The lane model of the intersection is established in VISSIM, and the hourly traffic volume and traffic composition of each approach investigated are incorporated into the model. Two-phase schemes, a symmetrical phase and a phase for each approach, are preliminarily proposed to judge whether the current signal control scheme is reasonable. The VISSIM simulation results show that the delay of the symmetrical phase is lower than that of a phase for each approach when a fixed signal control scheme is used at the intersection. Therefore, the symmetrical phase currently in 
operation at the intersection is generally feasible, and the current signal timing parameters do not waste green time in the approach with the largest traffic volume.

\subsection{Simulation Process}

3.3.1. Calibration of VISSIM Parameters. In the process of verifying whether the current signal control scheme is reasonable, all parameters in VISSIM adopt default values. In order to keep the simulation results consistent with the actual operation results, the model parameters in VISSIM need to be corrected [40].

First, the saturation flow rate of each lane is investigated, and the mean speed and current signal timing parameters are incorporated into the simulation model as the initial correction values. It is found that the mean error of delay, queue length, and stop rate between the simulation results and the actual investigation results are approximately $11 \%$. Then, the lane saturation flow rate, car-following model parameters, minimum spacing, and values of acceleration and deceleration are adjusted by small steps until the simulation results are essentially consistent with the actual investigation results with a mean error within 3\%. At this point, the calibration of the VISSIM simulation parameters is considered complete.

The calibration results are as follows: the saturation flow rates of the left-turn and through lanes are $1400 \mathrm{pcu} / \mathrm{h}$ and $1550 \mathrm{pcu} / \mathrm{h}$, respectively, the minimum spacing is $1.0 \mathrm{~m}$, the maximum acceleration (deceleration) is $4.5 \mathrm{~m} / \mathrm{s}^{2}$, the expected acceleration (deceleration) is $3.0 \mathrm{~m} / \mathrm{s}^{2}$, the average standstill distance is $1.5 \mathrm{~m}$, the minimum headway is $0.5 \mathrm{~m}$, the number of observed vehicles is 2 vehicles, the look ahead distance is $200 \mathrm{~m}$, the additive part of the safety distance is 2.0, and the multiplicative part of the desired safety distance is 3.0. The results of the simulation are shown in Tables 3-5.

3.3.2. Establishment of the Simulation Environment. The signal control method proposed in this study is programmed using the dynamic link library. Through the API interface in VISSIM, the customized signal control method is connected to the VISSIM traffic light controller [40]. Two sets of traffic flow detectors are laid at each approach in accordance with the results of $L^{d_{1}}$ and $L^{d_{2}}$ in Table 2 .

In the simulation process, the state of each detector is collected every $0.5 \mathrm{~s}$ to obtain the traffic volume of arrivals and departures and whether the traffic flow is saturated when the queue traffic flow dissipates during the green time. Then, according to the rules in Sections 2.3 and 2.5, the phase scheme and signal timing scheme of each cycle is determined. The asymmetrical threshold value of each opposite traffic flow is 0.2 and $l=3 \mathrm{~s}$.

In order to reproduce the asymmetry of the opposite traffic flow at the investigated intersection in VISSIM, the vehicle generator is adjusted every 10 seconds. The adjustment is based on the investigated traffic volume at the intersection in all approaches per 10 seconds [40].

In order to comprehensively evaluate the advantages and disadvantages of the optimization signal control scheme proposed in this study, the average delay per vehicle, average queue length, and stop rate are selected as evaluation indexes. An induced signal control scheme and an approximate dynamic programming (ADP) scheme by Cai et al. [20] are added for comparative analysis. ADP is an adaptive traffic signal controller for real-time operations. The working principle of ADP is similar to that of the method proposed in this study by changing the phase scheme and signal timing according to the dynamical traffic flow of each approach. The difference is that the phase scheme is one phase for each approach, and the release order of the phases and whether each phase is executed or not in each cycle are variable in ADP. ADP does not consider whether the opposite traffic flow for the opposite approaches is symmetrical or not.

In addition, the impact of an accident on traffic signal control has received special attention in recent years $[7,41]$. Therefore, two traffic scenarios are designed in this paper, as shown in Figure 6. In the first scenario, an accident is occurring at the upstream of the west approach, which will affect the vehicles entering the west approach. In the second scenario, an accident is occurring at the downstream of east exit, which will affect the vehicles driving out of east exit. We assume that the accident will result in the closure of two lanes on the road segment. Compared with the method proposed in reference [40], it is used to verify the ability of the method proposed in this study to respond to an accident.

The phase scheme for induced signal control is the same as that for fixed signal control. The initial green time and maximum green extension time of each phase are 0.5 and 1.5 times the green time of the corresponding fixed signal control scheme, respectively, and the unit extension time is $3 \mathrm{~s}$.

3.3.3. Simulation Steps. Under the condition that the calibrated simulation parameters and the input traffic volume in each approach remain unchanged, a traffic simulation of the intersection is conducted repeatedly in accordance with the three types of signal control schemes and two traffic scenarios. Each simulation lasted for 30 minutes.

\section{Results and Discussion}

4.1. Results Analysis. The simulation results are shown in Tables 6-9. In the half hour traffic simulation of the method proposed in this study, eight out of 17 signal cycles adopted the overlapping phase, one cycle adopted a phase for each approach, and the other eight cycles adopted the completely symmetrical phase.

A comparison of the simulation results in Tables 3 and 6 shows that the optimization method proposed in this study reduces the average delay per vehicle from $63.3 \mathrm{~s}$ to $45.6 \mathrm{~s}$, which correspond to a decrease of $28 \%$ compared to the current scheme under the same traffic conditions. In terms of the direction of traffic flow and each approach, the decrease in delay before and after the improvement is also significant. The decrease in delay for the through traffic flow is about $30 \%$, while that for the left-turn traffic flow is 
TABLE 3: Simulation delay of the current intersection signal control scheme.

\begin{tabular}{|c|c|c|c|c|c|c|c|c|}
\hline \multirow{2}{*}{ Items } & \multicolumn{2}{|c|}{ South approach } & \multicolumn{2}{|c|}{ West approach } & \multicolumn{2}{|c|}{ North approach } & \multicolumn{2}{|c|}{ East approach } \\
\hline & Left-turn & Through & Left-turn & Through & Left-turn & Through & Left-turn & Through \\
\hline Flow (s) & 47.2 & 85.3 & 46.7 & 56 & 41.5 & 52.2 & 49.1 & 71 \\
\hline Approach (s) & \multicolumn{2}{|c|}{76.6} & \multicolumn{2}{|c|}{54.6} & \multicolumn{2}{|c|}{50.8} & \multirow{2}{*}{\multicolumn{2}{|c|}{67.5}} \\
\hline Intersection $(\mathrm{s})$ & & & & & & & & \\
\hline
\end{tabular}

TABLE 4: Simulation queue length of the current intersection signal control scheme.

\begin{tabular}{|c|c|c|c|c|c|c|c|c|c|}
\hline & \multirow{2}{*}{ Items } & \multicolumn{2}{|c|}{ South pproach } & \multicolumn{2}{|c|}{ West approach } & \multicolumn{2}{|c|}{ North approach } & \multicolumn{2}{|c|}{ East approach } \\
\hline & & Left-turn & Through & Left-turn & Through & Left-turn & Through & Left-turn & Through \\
\hline Average & $\begin{array}{c}\text { Flow }(\mathrm{m}) \\
\text { Intersection }(\mathrm{m})\end{array}$ & 13 & 88 & 8 & 33 & 16 & 47 & 10 & 58 \\
\hline
\end{tabular}

TABLE 5: Simulation stop rate of the current signal control scheme at the intersection.

\begin{tabular}{lcccccccc}
\hline \multirow{2}{*}{ Items } & \multicolumn{2}{c}{ South approach } & \multicolumn{2}{c}{ West approach } & \multicolumn{2}{c}{ North approach } & \multicolumn{2}{c}{ East approach } \\
& Left-turn & Through & Left-turn & Through & Left-turn & Left-turn & Through & Left-turn \\
\hline Number of stops (veh) & 32 & 160 & 19 & 125 & 24 & 200 & 23 \\
Vehicles of pass through (veh) & 43 & 146 & 21 & 123 & 29 & 201 & 24 \\
Stop rate & 0.74 & 1.10 & 0.90 & 1.02 & 0.83 & 1.00 & 0.99 & 1.65 \\
Approach & & 1.02 & & 1 & & & \\
Intersection & & & & & & & & \\
\end{tabular}

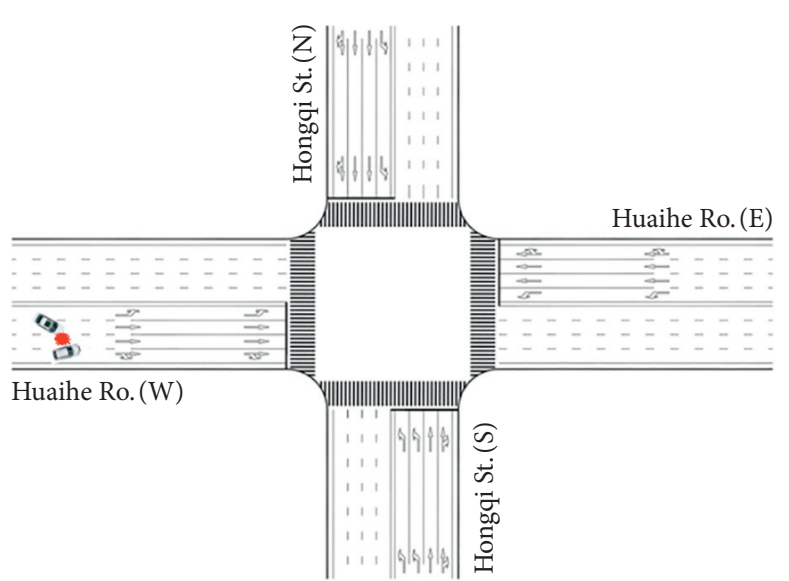

(a)

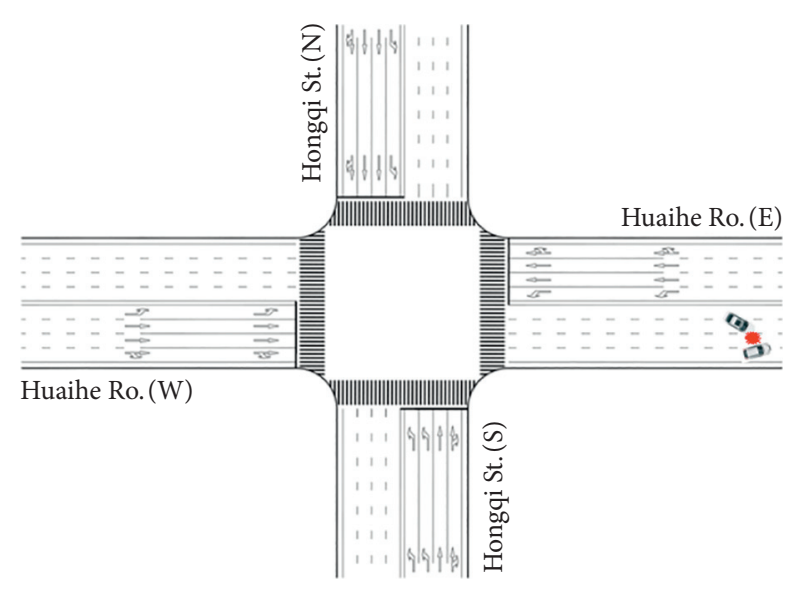

(b)

Figure 6: Two scenarios incident related. (a) An accident at upstream of the west approach. (b) An accident at downstream of the east exit.

Table 6: Delay of the Hongqi Street and Huaihe Road intersection after optimization.

\begin{tabular}{|c|c|c|c|c|c|c|c|c|}
\hline \multirow{2}{*}{ Items } & \multicolumn{2}{|c|}{ South approach } & \multicolumn{2}{|c|}{ West approach } & \multicolumn{2}{|c|}{ North approach } & \multicolumn{2}{|c|}{ East approach } \\
\hline & Left-turn & Through & Left-turn & Through & Through & Left-turn & Through & Left-turn \\
\hline Flow (s) & 41.3 & 60.7 & 37.6 & 38.1 & 38.4 & 38.5 & 40.6 & 50.8 \\
\hline $\begin{array}{l}\operatorname{Approach}(\mathrm{s}) \\
\text { Intersection }(\mathrm{s})\end{array}$ & \multicolumn{2}{|c|}{54.9} & \multicolumn{2}{|c|}{38.7} & \multicolumn{2}{|c|}{41.2} & \multicolumn{2}{|c|}{48.4} \\
\hline
\end{tabular}

smaller, ranging from $7.5 \%$ to $17.3 \%$. The extent of the delay decrease in various traffic flow directions is closely related to the traffic volume in each approach. The larger the traffic volume, the more the delays will be saved when the overlapping phase is used and the faster the delay will decrease. The key to achieving a conspicuous decrease in the average 
TABLe 7: Queue length of the Hongqi Street and Huaihe Road intersection after optimization.

\begin{tabular}{lccccccccc}
\hline & \multirow{2}{*}{ Items } & \multicolumn{2}{c}{ South approach } & \multicolumn{2}{c}{ West approach } & \multicolumn{2}{c}{ North approach } & \multicolumn{2}{c}{ East approach } \\
& & Left-turn & Through & Left-turn & Through & Left-turn & Left-turn & Through & Left-turn \\
\hline \multirow{2}{*}{ Average } & Flow $(\mathrm{m})$ & 10 & 60 & 6 & 20 & 12 & 31 & 7 \\
& Intersection $(\mathrm{m})$ & & & & & 22 & & & 28 \\
\hline
\end{tabular}

Table 8: Stop rate of the Hongqi Street and Huaihe Road intersection after optimization.

\begin{tabular}{|c|c|c|c|c|c|c|c|c|}
\hline \multirow{2}{*}{ Items } & \multicolumn{2}{|c|}{ South approach } & \multicolumn{2}{|c|}{ West approach } & \multicolumn{2}{|c|}{ North approach } & \multicolumn{2}{|c|}{ East approach } \\
\hline & Left-turn & Through & Left-turn & Through & Left-turn & Left-turn & Through & Left-turn \\
\hline Number of stops (veh) & 33 & 151 & 22 & 110 & 29 & 210 & 25 & 158 \\
\hline Vehicles of pass through (veh) & 46 & 173 & 34 & 145 & 38 & 228 & 38 & 165 \\
\hline Stop rate & 0.71 & 0.87 & 0.64 & 0.76 & 0.76 & 0.92 & 0.66 & 0.96 \\
\hline Approach & \multicolumn{2}{|c|}{0.79} & \multicolumn{2}{|c|}{0.70} & \multicolumn{2}{|c|}{0.84} & \multicolumn{2}{|c|}{0.81} \\
\hline Intersection & \multicolumn{8}{|c|}{0.78} \\
\hline
\end{tabular}

TABLE 9: Comparison of various signal control schemes.

\begin{tabular}{|c|c|c|c|c|c|c|}
\hline \multicolumn{2}{|l|}{ Items } & $\begin{array}{l}\text { Average delay } \\
\text { (s) }\end{array}$ & Saturation & $\begin{array}{l}\text { Average queue length } \\
(\mathrm{m})\end{array}$ & $\begin{array}{l}\text { Maximum queue length } \\
(\mathrm{m})\end{array}$ & $\begin{array}{l}\text { Stop } \\
\text { rate }\end{array}$ \\
\hline \multicolumn{2}{|l|}{ Induced scheme } & 59.7 & 0.76 & 32 & 97 & 1.03 \\
\hline \multicolumn{2}{|l|}{$\mathrm{ADP}[20]$} & 53.9 & 0.74 & 29 & 94 & 0.92 \\
\hline \multicolumn{2}{|c|}{ Optimization scheme in this study } & 45.6 & 0.70 & 22 & 86 & 0.78 \\
\hline \multirow{2}{*}{$\begin{array}{l}\text { An accident at } \\
\text { approach }\end{array}$} & Reference $[41]$ & 53.7 & 0.73 & 28 & 95 & 0.91 \\
\hline & $\begin{array}{l}\text { Optimization scheme in this } \\
\text { study }\end{array}$ & 43.9 & 0.68 & 20 & 81 & 0.76 \\
\hline \multirow[b]{2}{*}{ An accident at exit } & Reference $[41]$ & 67.3 & 0.92 & 42 & 118 & 1.13 \\
\hline & $\begin{array}{l}\text { Optimization scheme in this } \\
\text { study }\end{array}$ & 78.3 & 1.02 & 62 & 177 & 1.41 \\
\hline
\end{tabular}

delay per vehicle is to use the alternating asymmetry of the arrival vehicles for releasing the through traffic flow in the approach with large traffic volume in advance. This results in a reduction in the waiting time of through vehicles and avoids the consumption of space-time resources on some approaches of the intersection.

It can be seen in Tables 4 and 7 that the average queue length is reduced from $34 \mathrm{~m}$ to $22 \mathrm{~m}$, which corresponds to a decrease of $35.3 \%$. In the direction of traffic flow, the drop in through traffic flow is more obvious than for leftturn traffic flow. The key to the decrease is that the use of overlapping phases shortens the waiting red time, such as for through traffic flow. At the same time, it also shortens the green time for the opposite release of subsequent through traffic flow, which makes the green light for the next left-turn traffic flow open in advance and also makes the queue length of the left-turn traffic flow decrease to a certain extent.

The use of overlapping phases closes the green light in the approach where the left-turn traffic volume is small in advance and increases the number of stops, as seen in Tables 5 and 8 . However, the use of overlapping phases increases the number of vehicles passing through the intersection. The simulation results show that the growth rate of the number of stops is slower than for the number of vehicles passing through, which reduces the total stop rate from 1.08 to 0.78 , dropped by $27.8 \%$.
Compared with the current fixed signal control scheme, the induced signal control scheme's advantages are not significant, as shown in Table 9. Although the traffic flow on the opposite approaches displays an alternately asymmetric state, the larger traffic volume change in the two approaches in each cycle is not significant, which is the key to deciding the green time of each phase by the induced signal control. The induced signal control also leads to green time waste in approaches with low traffic volume.

ADP is a better choice than induced signal control because it can optimize the phase sequence. The phase sequence of five cycles changes in 17 signal cycles. The simulation results of the ADP are not as good as those of the method proposed in this study, which is closely related to the geometric size and traffic volume of the selected intersection. The traffic volume of the chosen intersection is large, and although the efficiency of the signal control can be improved by optimizing the phase sequence, each phase in each cycle must be executed, and there is no possibility of skipping any phase. In addition, the adaptability of a fixed number of phases to asymmetric traffic flow is relatively poor. According to the results presented in [24], ADP has an improved control effect on low saturation intersections.

As can be seen from Table 9, when an accident is occurring at the upstream of the west approach, the vehicles entering the west approach are reduced because two lanes are occupied by the accident, which aggravates the 
asymmetry of traffic flows between east approach and west approach. The symmetrical phase used in reference [41] will lead to a low utilization rate of the green time on the west approach. However, the method proposed in this paper adopts the scheme of a phase for east approach and a phase for west approach in more and more cycles, and it is more beneficial to the optimal utilization of the intersection spacetime resources.

When an accident is occurring at the downstream of east exit, two exit lanes are also occupied due to the accident, resulting in the obstruction of the vehicles running out of east exit, and the queue gradually spreads to the intersection, which will eventually lead to the traffic congestion in the intersection. The method proposed in reference [41] strengthens the detection of accidents at all approaches and exits. Once an accident is found, it will restrain the vehicles entering the accident-related exit. Although the delays at the approaches are also increased, it will not block the intersection, and the signal control strategy is more effective than the method presented in this paper. Therefore, the method proposed in this paper has a weak adaptive adjustment ability to exit accident because it does not consider the realtime traffic status detection of exits.

\subsection{Sensitivity Analysis}

4.2.1. The Influence of a Changing Asymmetric Coefficient on the Delay of Opposite Approaches. Let the sum of the leftturn volumes and through traffic volumes of the east and west approaches for low, medium, and high traffic volumes be $400 \mathrm{pcu} / \mathrm{h}, 800 \mathrm{pcu} / \mathrm{h}$, and $1200 \mathrm{pcu} / \mathrm{h}$, respectively. The left-turn traffic volume of $220 \mathrm{pcu} / \mathrm{h}$ and the through traffic volume of $800 \mathrm{pcu} / \mathrm{h}$ for the north-south approach remain unchanged. When the ratio of the sum of the left-turn traffic volume and the sum of the through traffic volume in the east-west approach is $1: 3,1: 4,1: 5$, and $1: 6$, adjusting the asymmetric coefficient of the left-turn vehicles, the average delay per vehicle for the east and west approaches is obtained through simulation by symmetrical release or east and west approach release separately. The average delay is shown in Figure 7.

It can be seen from Figure 7 (a) that, in a low traffic volume environment, due to the minimum green time constraints in each phase, the influence of the asymmetric coefficients on the release mode of the traffic flow and average delay is weak. However, with an increase in traffic volume, the change in the asymmetric coefficient has a more obvious influence on the release mode and average delay. This indicates that the greater the asymmetric coefficient, the lower the efficiency of the symmetrical release phase scheme. In addition, a greater asymmetric coefficient is more unfavorable to the balanced use of the space-time resources at intersections.

Under the same traffic volume, with an increase in the asymmetric coefficient, the average delay per vehicle for the symmetrical release increases rapidly, especially in medium and high traffic volumes. When the asymmetric coefficient exceeds a critical value, the average delay of the symmetrical release is greater than that of the independent release for the single approach. This can be seen clearly in Figures 7(b) and 7(c). This behavior shows that when the equilibrium state of the opposite traffic flow in one phase changes, the benefits of the symmetrical release phase scheme are weakened, while the independent release of the single approach is beneficial to the balanced utilization of space-time resources at intersections. However, when the asymmetric state of opposite traffic flow is not significant, symmetrical release is still an effective form of signalized control. Only when the asymmetric state is significant will the symmetrical release fail. It can then be concluded that the symmetric release phase scheme for asymmetric traffic flow needs to meet certain constraints.

When the ratio of the sum of left-turn traffic volume to the sum of through traffic volume is different, the critical value of the asymmetric coefficient corresponding to the conversion of the benefit level under different release modes is also different.

\subsubsection{Influence of the Asymmetric Coefficient Threshold on} Phase Delay. The influence of a change in the asymmetry coefficient threshold on the phase delay is analyzed under the condition that the left-turn and through traffic volumes and the asymmetry coefficient threshold remain unchanged for the south and north approaches. The left-turn traffic volume is fixed, and its asymmetric coefficient in the eastwest approach is given the value of $a c_{1}=0.2$. At the same time, the sum of the traffic volume of the through phase in the east-west approach is unchanged. Then, the asymmetric coefficient of the through phase in the east-west approach is adjusted from 0.1 to 0.5 using a step size of 0.1 (i.e., $a c_{2}=0.1$, $0.2,0.3,0.4$, and 0.5 ). Using simulations, the average delay per vehicle in the through phase in the east-west approach is obtained under various threshold conditions $\left(a c_{1,0}=a c_{2,0}=0.1, \ldots, 0.7\right)$, as shown in Figure 8 . The selection of a threshold ranging from 0.1 to 0.7 is proposed to determine which point is the critical transition point for the two types of phase schemes by the simulation delays within the threshold ranges. Therefore, the threshold range is made relatively large.

As seen in Figure 8(a), as the asymmetric coefficient threshold increases, the average delay of the through phase increases, with the increase being significant when the threshold is greater than 0.2 . The asymmetry coefficient threshold dictates the release mode of opposite traffic flow. Therefore, even though the average single-lane traffic volume of the east and west approaches differs greatly when the asymmetric coefficient threshold is higher, the opposite traffic flow is judged to be symmetric and released symmetrically. Owing to an increase in the traffic flow ratio of the critical lane group, the green time and cycle in this phase will increase, which will inevitably lead to an increase in the average delay. It can be concluded from Figure 8(b) that an asymmetric coefficient threshold of 0.2 is reasonable for the through phase, and calibration of the asymmetric coefficient threshold in the other phases is the same. 


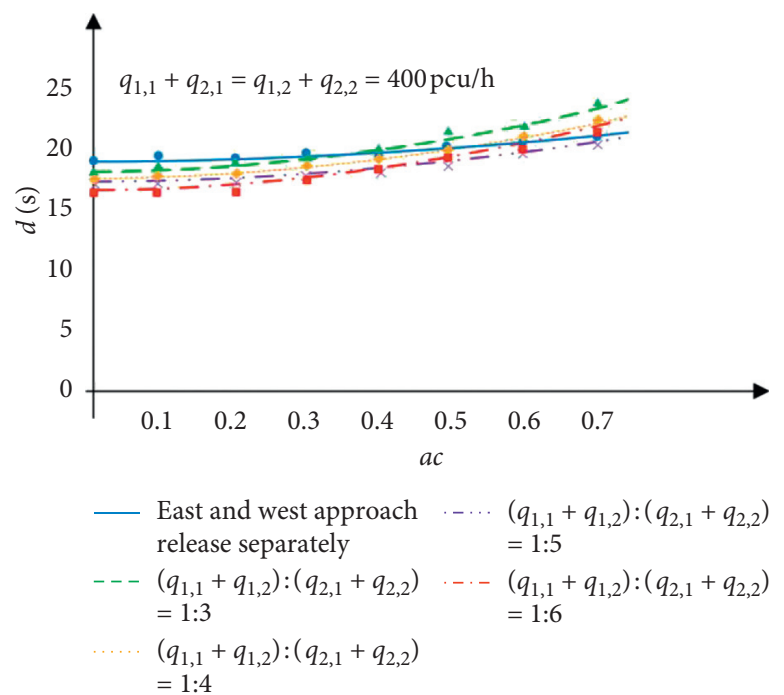

(a)

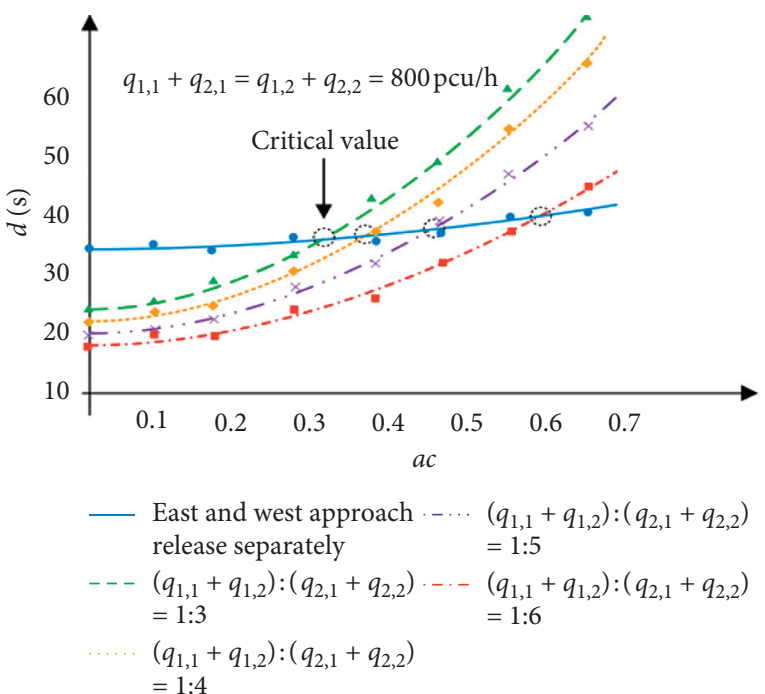

(b)

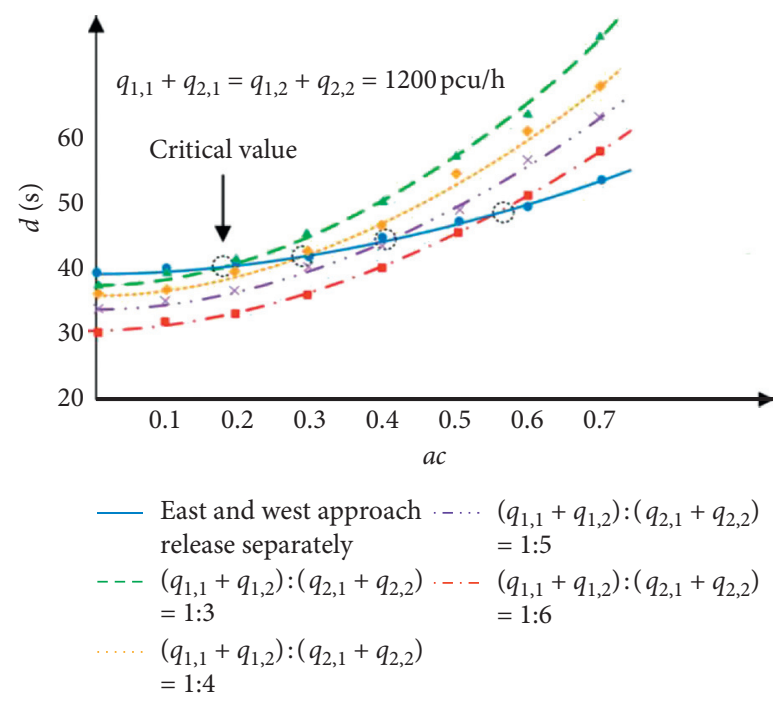

(c)

FiguRe 7: Simulation results of average delay versus changing asymmetric coefficient under different traffic volumes. (a) Average delay under low traffic volume. (b) Average delay under medium traffic volume. (c) Average delay under high traffic volume.

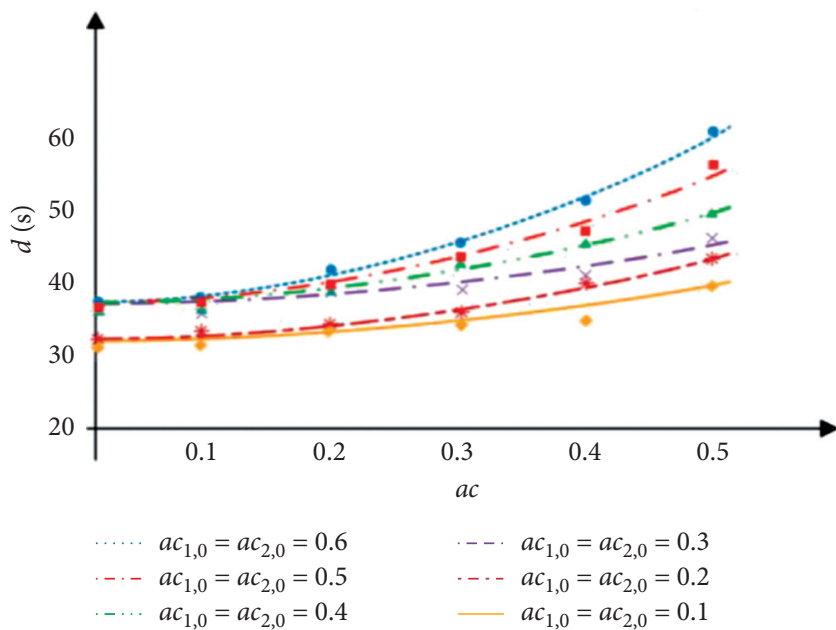

(a)

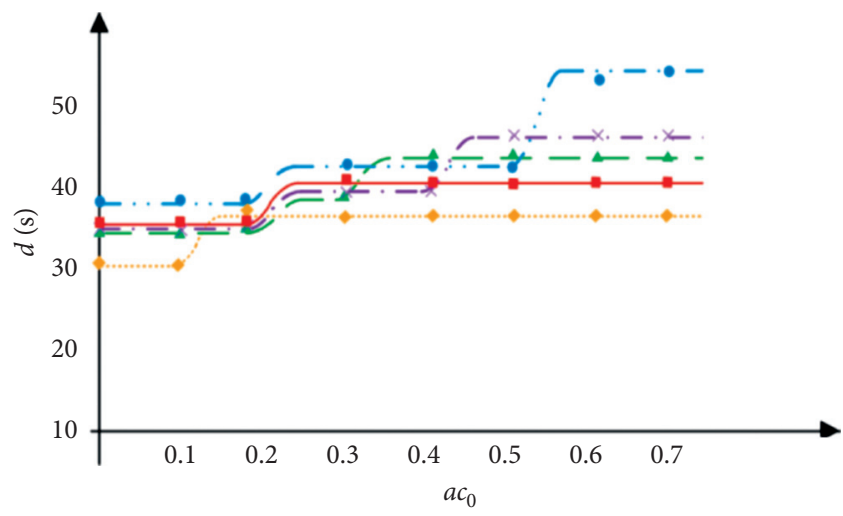

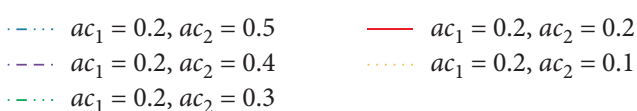

(b)

Figure 8: Asymmetric coefficient threshold on the phase delay. (a) Variation of phase delay with the asymmetric coefficient under different thresholds. (b) Variation of phase delay with threshold under different asymmetric coefficients. 


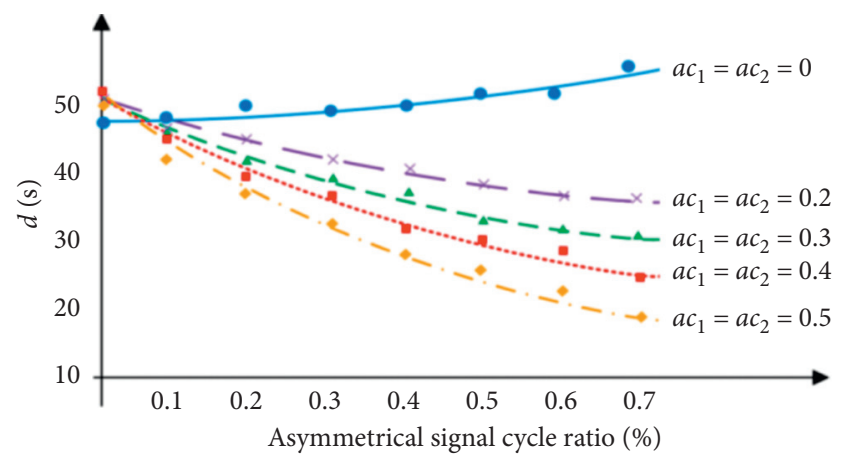

Figure 9: Asymmetric signal cycle ratio on the phase delay.

4.2.3. Influence of the Asymmetrical Signal Cycle Ratio on Intersection Delay. An asymmetric signal cycle means that the opposite traffic flow of one or more phases in the cycle is in an asymmetric state. The sum of left-turn and through traffic volumes in the south-north approach is held unchanged, and $a c_{3}=a c_{4}$. The asymmetric coefficients of the east-west left-turn phase and the east-west through phase in the asymmetric signal cycle are taken as $0.2,0.3,0.4$, and 0.5 , and the proportion of the asymmetric signal cycle in the total cycles within one hour is taken as $10 \%, 20 \%, 30 \%, 40 \%, 50 \%$, $60 \%$, and $70 \%$. The dynamic phase scheme is selected according to Section 2.3.2, and the cycle is redefined per signal cycle. The average delay per vehicle at the intersection by the delay model is calculated as shown in Figure 9.

As can be seen in Figure 9, with the increasing proportion in the asymmetric signal cycle, the average delay at the intersection demonstrates a decreasing trend. The optimized signal control scheme considers the change in the traffic flow operational state, reintegrates the traffic flow without conflict at the intersection, and dynamically generates a new signal control scheme. This reduces unnecessary waiting time for the traffic flow in some approaches, which is an improvement over the conventional symmetrical signal control method. In addition, when the proportion of the asymmetrical signal cycle is the same, the higher the asymmetric coefficient, the faster the delay decreased. This occurs because the conventional symmetric signal control method is less applicable at higher asymmetric coefficients, while the optimization method can integrate the space-time resources of different approaches at the intersection, consequently reducing the green time and average delay.

\section{Conclusions}

A dynamic phase signal control method is presented for unstable asymmetric traffic flow in this paper. Comparing to the existing work, our contributions mainly are that (1) we propose the generation rules for the dynamic phase scheme based on unstable asymmetric traffic flow per cycle, (2) we build the delay variation models of intersections adopting the dynamic phase scheme, and (3) we construct the adjustment algorithm of signal control parameters for the dynamic phase scheme.

VISSIM simulation shows that the optimization method proposed in this study can reduce the average vehicle delay by $28 \%$, the average queue length by $35.3 \%$, and the stop rate by $27.8 \%$, which demonstrates that the optimization method is effective. It is found that a higher asymmetric coefficient leads to lower efficiency of a symmetrical release phase scheme and is more unfavorable to the balanced use of space-time resources at intersections. With an increase in the asymmetric coefficient threshold, the average delay increases, and the increase becomes significant when the threshold is greater than 0.2 . With an increase in the proportion of the asymmetric signal cycle, the average delay at the intersection demonstrates a decreasing trend. For a similar proportion of asymmetrical signal cycles, a higher asymmetric coefficient leads to a faster delay decrease.

The method proposed in this paper does not consider the real-time traffic status detection of exits, and it has a weak adaptive adjustment ability to exit accident. The method also neglects the vehicular movement inside the intersection [42] and other travelers' acceptability, such as bicycles and pedestrians, and they may feel confused about the switch of the signal timing. In addition, for the intersection where the opposite traffic flow is stable and symmetrical, the proposed method degrades to the general signal control method for fixed phases and phase sequences. Therefore, the method is suitable for intersections with unstable opposite traffic flow.

\section{Data Availability}

The data used to support the findings of this study are presented in the case study of the manuscript.

\section{Conflicts of Interest}

The authors declare that they have no conflicts of interest regarding the publication of this paper.

\section{Acknowledgments}

This research was supported by the Harbin Special Fund Program in Innovation Talents of Science and Technology (No. 2016RAQXJ079).

\section{Supplementary Materials}

The other original data used in this paper has been uploaded by attachment. (Supplementary Materials)

\section{References}

[1] K. Wada, K. Usui, T. Takigawa, and M. Kuwahara, "An optimization modeling of coordinated traffic signal control based on the variational theory and its stochastic extension," Transportation Research Part B: Methodological, vol. 117, pp. 907-925, 2018.

[2] H. Yu, R. Ma, and H. M. Zhang, "Optimal traffic signal control under dynamic user equilibrium and link constraints in a general network," Transportation Research Part B: Methodological, vol. 110, pp. 302-325, 2018.

[3] C. X. Ma and P. F. Liu, "Intersection signal timing optimization considering the travel safety of the elderly," Advances in Mechanical Engineering, vol. 11, no. 12, pp. 1-8, 2019.

[4] S.-W. Chiou, "A robust signal control system for equilibrium flow under uncertain travel demand and traffic delay," Automatica, vol. 96, pp. 240-252, 2018. 
[5] H. Wei, L. B. Li, and H. K. Lo, "Adaptive traffic signal control with equilibrium constraints under stochastic demand," Transportation Research Part C, vol. 95, pp. 394-413, 2018.

[6] C. Ma, J. Zhou, X. Xu, and J. Xu, "Evolution regularity mining and gating control method of urban recurrent traffic congestion: a literature review," Journal of Advanced Transportation, vol. 2020, Article ID 5261580, 13 pages, 2020.

[7] L. Qi, M. C. Zhou, and W. J. Luan, “A two-level traffic light control strategy for preventing incident-based urban traffic congestion," IEEE Transactions on Intelligent Transportation Systems, vol. 19, no. 1, pp. 13-24, 2017.

[8] C. Ma and R. He, "Green wave traffic control system optimization based on adaptive genetic-artificial fish swarm algorithm," Neural Computing and Applications, vol. 31, no. 7, pp. 2073-2083, 2019.

[9] S. Zhou, W. Liang, J. Li, and J.-U. Kim, "Improved VGG model for road traffic sign recognition," Computers, Materials \& Continua, vol. 57, no. 1, pp. 11-24, 2018.

[10] B.-L. Ye, W. Wu, K. Ruan et al., "A survey of model predictive control methods for traffic signal control," IEEE/CAA Journal of Automatica Sinica, vol. 6, no. 3, pp. 623-640, 2019.

[11] W. Liu, Y. Xu, J. C. N. Yang, W. Yu, and L. Chi, "Privacypreserving quantum two-party geometric intersection," Computers, Materials \& Continua, vol. 60, no. 3, pp. 12371250, 2019.

[12] L. Li, Y. S. Lv, and F. Y. Wang, "Traffic signal timing via deep reinforcement learning," IEEE/CAA Journal of Automatica Sinica, vol. 3, no. 3, pp. 247-254, 2016.

[13] B. Mohammed and D. Naouel, "An efficient greedy traffic aware routing scheme for internet of vehicles," Computers, Materials \& Continua, vol. 60, no. 3, pp. 959-972, 2019.

[14] T. Le, P. Kovács, N. Walton, H. L. Vu, L. L. H. Andrew, and S. S. P. Hoogendoorn, "Decentralized signal control for urban road networks," Transportation Research Part C: Emerging Technologies, vol. 58, pp. 431-450, 2015.

[15] M. Abbas, D. Bullock, and L. Head, "Real-time offset transitioning algorithm for coordinating traffic signals," Transportation Research Record: Journal of the Transportation Research Board, vol. 1748, no. 1, pp. 26-39, 2001.

[16] P. Mirchandani and L. Head, "A real-time traffic signal control system: architecture, algorithms, and analysis," Transportation Research Part C: Emerging Technologies, vol. 9, no. 6, pp. 415-432, 2001.

[17] H. Rene and V. W. Jan, "An MDP decomposition approach for traffic control at isolated signalized intersections," Probability in the Engineering and Informational Sciences, vol. 22, no. 4, pp. 587-602, 2008.

[18] S. J. Agbolosu-Amison, I. Yun, and B. B. Park, "Quantifying benefits of a dynamic gap-out feature at an actuated traffic signalized intersection under cooperative vehicle infrastructure system," KSCE Journal of Civil Engineering, vol. 16, no. 3, pp. 433-440, 2012.

[19] W. Ma, K. An, and H. K. Lo, "Multi-stage stochastic program to optimize signal timings under coordinated adaptive control," Transportation Research Part C: Emerging Technologies, vol. 72, pp. 342-359, 2016.

[20] C. Cai, C. K. Wong, and B. G. Heydecker, "Adaptive traffic signal control using approximate dynamic programming," Transportation Research Part C: Emerging Technologies, vol. 17, no. 5, pp. 456-474, 2009.

[21] L. Wang, M. Z. Wang, F. Zhou et al., "Modeling and analyzing of real-time adaptive traffic signal control with CPN," Journal of Highway and Transportation Research and Development, vol. 6, no. 25, pp. 115-119, 2008.
[22] W. Cheng, X. Liu, and W. Zhang, "An optimal adaptive traffic signal control algorithm for intersections group," in Proceedings of the World Congress on Intelligent Control and Automation, Dalian, China, June 2006.

[23] A. Ahmad and A. Naeem, "Optimized timing parameters for real-time adaptive traffic signal controller," in Proceedings of the IEEE International Computer Conference in Modelling and Simulation, Cambridge, UK, March 2014.

[24] B. Yin, A. El Moudni, and M. Dridi, "Forward search algorithm based on dynamic programming for real-time adaptive traffic signal control," IET Intelligent Transport Systems, vol. 9, no. 7, pp. 754-764, 2015.

[25] L. Gao, "The intelligent traffic control model of multiphase intersection with variable phase sequence," in Proceedings of the 2017 16th International Symposium on Distributed Computing and Applications to Business, Engineering and Science (DCABES), Anyang, China, November 2017.

[26] J. Zhao, W. Ma, H. M. Zhang, and X. Yang, "Two-step optimization model for dynamic lane assignment at isolated signalized intersections," Transportation Research Record: Journal of the Transportation Research Board, vol. 2355, no. 1, pp. 39-48, 2013.

[27] X. Li, H. Wang, and J. Chen, "Dynamic lane-use assignment model at signalized intersections under tidal flow," in Proceedings of the Fourth International Conference on Transportation Engineering, Chengdu, China, October 2013.

[28] M. W. Levin and S. D. Boyles, "A cell transmission model for dynamic lane reversal with autonomous vehicles," Transportation Research Part C: Emerging Technologies, vol. 68, pp. 126-143, 2016.

[29] K. J. Assi and N. T. Ratrout, "Proposed quick method for applying dynamic lane assignment at signalized intersections," IATSS Research, vol. 42, no. 1, pp. 1-7, 2018.

[30] A. Harvey and D. Bullock, "Implementation of a distributed control system for dynamic lane assignment," in Proceedings of the Southeastern Symposium on System Theory, Baton Rouge, LA, USA, March 1996.

[31] W. H. K. Lam, A. C. K. Poon, and G. K. S. Mung, "Integrated model for lane-use and signal-phase designs," Journal of Transportation Engineering, vol. 123, no. 2, pp. 114-122, 1997.

[32] C. K. Wong and S. C. Wong, "Lane-based optimization of signal timings for isolated junctions," Transportation Research Part B: Methodological, vol. 37, no. 1, pp. 63-84, 2003.

[33] Z. J. Zhong, H. D. Liu, W. J. Ma et al., "An optimization method of dynamic lane assignment at signalized intersection," in Proceedings of the International Conference on Intelligent Computation Technology and Automation (ICICTA), pp. 1277-1280, Changsha, China, October 2008.

[34] Y. Zeng, X. G. Yang, and Y. Y. Ma, "Study on synergy problem of dynamic lane-use assignment and signal control at intersections," Journal of Tongji University, vol. 37, no. 7, pp. 903-908, 2009.

[35] W. K. M. Alhajyaseen, N. T. Ratrout, K. J. Assi, and A. A. Hassan, "The integration of dynamic lane grouping technique and signal timing optimization for improving the mobility of isolated intersections," A rabian Journal for Science and Engineering, vol. 42, no. 3, pp. 1013-1024, 2017.

[36] G. Y. Wu, K. Boriboonsomsin, L. P. Zhang et al., "Simulationbased benefit evaluation of dynamic lane grouping strategies at isolated intersections," in Proceedings of the IEEE Conference on Intelligent Transportation Systems, Anchorage, AK, USA, September 2012.

[37] W. K. M. Alhajyaseen, M. Najjar, N. T. Ratrout, and K. Assi, "The effectiveness of applying dynamic lane assignment at all 
approaches of signalized intersection," Case Studies on Transport Policy, vol. 5, no. 2, pp. 224-232, 2017.

[38] J. Zhao, J. Yao, S. He, and Y. Han, "Operational efficiency evaluation of intersections with dynamic lane assignment using field data," Journal of Advanced Transportation, vol. 2017, Article ID 2130385, 13 pages, 2017.

[39] R. Akcelik, Traffic Signals: Capacity and Timing Analysis, Australian Road Research Board, Melbourne, Australia, 6th edition, 1995.

[40] X. Jiang and S. Gao, "Signal control method and performance evaluation of an improved displaced left-turn intersection design in unsaturated traffic conditions," Transportmetrica $B$ : Transport Dynamics, vol. 8, no. 1, pp. 264-289, 2020.

[41] L. Qi, M. C. Zhou, and W. J. Luan, "Emergency traffic-light control system design for intersections subject to accidents," IEEE Transactions on Intelligent Transportation Systems, vol. 17, no. 1, pp. 170-183, 2015.

[42] J. Zhao, V. L. Knoop, and M. Wang, "Two-dimensional vehicular movement modelling at intersections based on optimal control," Transportation Research Part B: Methodological, vol. 138, pp. 1-22, 2020. 